MATHEMATICS OF COMPUTATION

Volume 70, Number 234, Pages 853-861

S 0025-5718(00)01191-1

Article electronically published on February 23, 2000

\title{
THE EIGHT DIMENSIONAL OVOIDS OVER GF(5)
}

\author{
C. CHARNES AND U. DEMPWOLFF
}

\begin{abstract}
In this article we outline a computer assisted classification of the ovoids in an orthogonal space of the type $\Omega^{+}(8,5)$.
\end{abstract}

\section{INTRODUCTION}

Let $V$ be a nondegenerate orthogonal vector space over $G F(q)$. An ovoid $\mathcal{O}$ is a set of isotropic points having precisely one point in common with each maximal, totally singular $r$-space (here $r$ is the Witt index of $V$ ). If $V$ has dimension $2 n$, we denote $V$ by $\Omega^{+}(2 n, q)$ if $r=n$ and by $\Omega^{-}(2 n, q)$ if $r=n-1$. Therefore an ovoid in $\Omega^{+}(2 n, q)$ is a set of $q^{n-1}+1$ pairwise nonperpendicular isotropic points. Various families of ovoids in orthogonal spaces are known. Blokhuis and Moorhouse 1 proved nonexistence results for ovoids in certain orthogonal spaces; see also Thas [17.

An important unsolved conjecture in Galois geometries states that there are no ovoids on hyperbolic quadrics in ten or more dimensions. This was known to be true for $q=2$, and for $q=3$ it was verified by Shult [16. The first open case is $q=5$. By projecting a $(2 n+2)$-dimensional ovoid in an isotropic point not belonging to it, a $2 n$-dimensional ovoid, called a section, is obtained. Shult showed that none of the ovoids in $\Omega^{+}(8,3)$ could occur as sections of a putative ovoid in ten dimensions. Similarly, a classification of the ovoids in $\Omega^{+}(8,5)$ could play a role in resolving this conjecture for $q=5$.

Ovoids in $V=\Omega^{+}(6, q)$ are mapped via the Klein correspondence onto translation planes of order $q^{2}$; see for example [10, [13. The translation planes of order $q^{2}$, and thus ovoids in $\Omega^{+}(6, q)$, have been classified for $q=2,3,4,5,7$; in [3], [5], [8], and [14. However, the ovoids in $\Omega^{+}(8, q)$ have only been classified for $q=2,3$ (see [10]), and for $q=4$ by Gunawardena [9].

In this paper we describe a computer-aided classification of the ovoids in $\Omega^{+}(8,5)$. We find that there are only three ovoids in $\Omega^{+}(8,5)$ up to equivalence. These ovoids have been known ever since the $E_{8}$ root lattice construction of Conway, Kleidman and Wilson 4 appeared.

In what follows, $V$ denotes an orthogonal space of type $\Omega^{+}(8, q)$, and if necessary we specify $q=5$.

\section{The Ovoids IN $\Omega^{+}(8,5)$}

Two ovoids $\mathcal{O}$ and $\mathcal{O}^{\prime}$ are considered to be equivalent if and only if there is a semisimilarity $\gamma$ in $\Gamma O(V)$ with $\mathcal{O}^{\prime}=\mathcal{O} \gamma$. Note that $G O(V)=\Gamma O(V)$ if $q$ is a

Received by the editor January 2, 1998 and, in revised form, April 20, 1999.

2000 Mathematics Subject Classification. Primary 51E15, 68R05, 05B25.

(C)2000 American Mathematical Society 
TABLE I. The ovoids in $\Omega^{+}(8,5)$.

\begin{tabular}{|c|c} 
Ovoid & Automorphism group \\
\hline $\mathcal{O}_{E}$ & $W\left(E_{7}\right)=2 \times S p(6,2)$ \\
$\mathcal{O}_{U}$ & $\operatorname{Aut}\left(U_{3}(5)\right) \simeq P G U_{3}(5) \cdot 2$ \\
$\mathcal{O}_{S}$ & $S_{10}$
\end{tabular}

prime, as in our case. A semisimilarity is defined as a semilinear map $f$ which acts as $(u f, v f)=s(u, v)^{\sigma}$, where $\sigma$ is a fixed field automorphism and $s \neq 0$ is a fixed field element. If $\sigma$ is the identity, $f$ is a similarity. The automorphism group $\operatorname{Aut}(\mathcal{O})$ of an ovoid $\mathcal{O}$ is the group of all the equivalence maps of $\mathcal{O}$. Three equivalence classes of ovoids in the orthogonal space $\Omega^{+}(8,5)$ have been known for some time. Their representatives, $\mathcal{O}_{E}, \mathcal{O}_{U}, \mathcal{O}_{S}$, and their automorphism groups are listed in Table \ Cooperstein's ovoid $\mathcal{O}_{S}$, and the ovoid $\mathcal{O}_{E}$ are instances of the $E_{8}$ root lattice constructions of [4], and $\mathcal{O}_{U}$ is the unitary ovoid studied by Kantor in [10, 11.

In the remainder of this paper we outline the proof of the following theorem.

Theorem 1. Any ovoid in the orthogonal space $\Omega^{+}(8,5)$ is equivalent to either $\mathcal{O}_{E}, \mathcal{O}_{U}$, or $\mathcal{O}_{S}$.

We will follow a similar strategy which we used in our previous classifications of the translation planes of order 27 and 49. This is described in detail in [3] and [7]. Thus we have to solve two problems:

1. Determine all the starter sets, i.e., find partial ovoids $\mathcal{S}_{1}, \ldots, \mathcal{S}_{k}$ such that any ovoid in $\Omega^{+}(8,5)$ contains up to equivalence one of the $\mathcal{S}_{i}$ 's. On the other hand, the sizes of the starter sets have to be large enough so that it is feasible to compute their completions to full ovoids.

2. Once all completions $\mathcal{O}_{1}, \ldots, \mathcal{O}_{n}$ of $\mathcal{S}_{1}, \ldots, \mathcal{S}_{k}$ have been computed, an effective equivalence test is required to sort the completed ovoids into equivalence classes.

\section{INVARIANTS AND EQUIVALENCE}

We use the fingerprint (see 2]) and related invariants to distinguish the equivalence classes of ovoids.

Let $\mathcal{O}=\left\{\left\langle v_{1}\right\rangle, \ldots,\left\langle v_{k}\right\rangle\right\}$ be a partial ovoid in $V$. For $q$ an odd prime and $x \in G F(q)$, define

$$
[x]= \begin{cases}0 & \text { if } x=0 \\ \left(\frac{x}{p}\right) & (\text { the Legendre symbol) otherwise. }\end{cases}
$$

The sign matrix $S \in \mathbf{Z}^{k \times k}$ has entries $s_{i j}=\left[\left(v_{i}, v_{j}\right)\right]$ and the quadratic matrix $Q \in \mathbf{Z}^{k \times k}$ is defined as $Q=S S^{T}$. ( $S^{T}$ is the transpose of $S$.) Let $\bar{Q}$ be the multiset of the absolute values of the entries in $Q$. The elements in $\bar{Q}$ are integers in the range $[0 \ldots k]$. Let $a_{j}$ be the multiplicity of $j$ in $\bar{Q}$. Then the fingerprint $f(\mathcal{O})$ of $\mathcal{O}$ is defined to be

$$
f(\mathcal{O})=\left(a_{0}, \ldots, a_{k}\right) \in \mathbf{Z}^{k} .
$$

It is easy to see (cf. [2] or [7]) that $f(\mathcal{O})$ is independent of the particular set of isotropic points used to represent $\mathcal{O}$, and that $f(\mathcal{O})$ is an invariant of equivalence. 
Suppose that $\gamma$ is a similarity with multiplier $s$, i.e., $(v \gamma, w \gamma)=s(v, w)$ for $v, w \in V$. Clearly the map $x \mapsto s x$ is a similarity with multiplier $s^{2}$ which fixes every ovoid. Thus if two (partial) ovoids $\mathcal{O}$ and $\mathcal{O}^{\prime}$ are equivalent, then there is an orthogonal map $\gamma \in O(V)$ with $\mathcal{O}^{\prime}=\mathcal{O} \gamma$ or $\mathcal{O}^{\prime}=\mathcal{O} \gamma_{0} \gamma$, where $\gamma_{0}$ is a similarity whose multiplier is a nonsquare. If $\mathcal{O}^{\prime}=\mathcal{O} \gamma$, we say that the ovoids are orthogonally equivalent.

Suppose that $\mathcal{O}=\left\{\left\langle v_{1}\right\rangle, \ldots,\left\langle v_{k}\right\rangle\right\}$ and $\mathcal{O}^{\prime}=\left\{\left\langle w_{1}\right\rangle, \ldots,\left\langle w_{k}\right\rangle\right\}$ are orthogonally equivalent. If $\left\{v_{1}, \ldots, v_{m}\right\}$ are linearly independent, $\gamma \in O(V)$ with $\mathcal{O}^{\prime}=\mathcal{O} \gamma$ and $\left\langle v_{i}\right\rangle \gamma=\left\langle w_{i}\right\rangle$ for $1 \leq i \leq k$, then the following properties hold:

1. $f\left(\mathcal{O}-\left\{\left\langle v_{1}\right\rangle, \ldots,\left\langle v_{m}\right\rangle\right\}\right)=f\left(\mathcal{O}^{\prime}-\left\{\left\langle w_{1}\right\rangle, \ldots,\left\langle w_{m}\right\rangle\right\}\right)$.

2. There exist scalars $\alpha_{1}, \ldots, \alpha_{m}$ in $G F(q)$ such that $\operatorname{Gr}\left(v_{1}, \ldots, v_{m}\right)=$ $\operatorname{Gr}\left(\alpha_{1} w_{1}, \ldots, \alpha_{m} w_{m}\right)$, where $\operatorname{Gr}(\ldots)$ denotes the Gram matrix of the respective $m$-tuples of vectors.

If however $\gamma$ is a similarity with multiplier $s$ and $\left\langle v_{i}\right\rangle \gamma=\left\langle w_{i}\right\rangle$ for $1 \leq i \leq k$, then the first property still holds but the second may fail. For instance if $\left\langle v_{1}, \ldots, v_{m}\right\rangle$ is nondegenerate, $m$ is odd, and $s$ is a nonsquare, then $\left\langle v_{1}, \ldots, v_{m}\right\rangle$ and $\left\langle w_{1}, \ldots, w_{m}\right\rangle$ have different discriminants. However in such a case we know that $\mathcal{O}^{-}=\mathcal{O} \gamma_{0}$ and $\mathcal{O}^{\prime}$ are orthogonally equivalent. So that for the pair $\left(\mathcal{O}^{-}, \mathcal{O}^{\prime}\right)$ we can find a $\gamma \in O(V)$ satisfying both properties.

Finally if $\left\langle v_{1}, v_{2}, v_{3}\right\rangle$ and $\left\langle w_{1}, w_{2}, w_{3}\right\rangle$ have the same discriminant, then there are scalars $\alpha_{1}, \alpha_{2}, \alpha_{3}$, which are unique up to sign, so that $\operatorname{Gr}\left(v_{1}, v_{2}, v_{3}\right)=$ $\operatorname{Gr}\left(\alpha_{1} w_{1}, \alpha_{2} w_{2}, \alpha_{3} w_{3}\right)$. We shall see later that the ovoids in $\Omega^{+}(8,5)$ always contain a basis, i.e., they generate the 8 -dimensional space $V$. Collecting these observations, we obtain the following algorithm for equivalence.

\section{EQUIVALENCE TEST}

INPUT: Ovoids $\mathcal{O}^{+}=\left\{\left\langle v_{1}\right\rangle, \ldots,\left\langle v_{126}\right\rangle\right\}, \mathcal{O}^{-}=\left\{\left\langle u_{1}\right\rangle, \ldots,\left\langle u_{126}\right\rangle\right\}, \mathcal{O}^{\prime}=\left\{\left\langle w_{1}\right\rangle\right.$, $\left.\ldots,\left\langle w_{126}\right\rangle\right\}$ such that $\left\{v_{1}, \ldots, v_{8}\right\}$ is a basis of $\Omega^{+}(8,5)$ and $u_{i}=v_{i} \gamma_{0}$ for $1 \leq i \leq$ 126 , where $\gamma_{0}$ is a fixed similarity whose multiplier is a nonsquare.

OUTPUT: $\mathrm{NO}$, if $\mathcal{O}^{\prime}$ is not equivalent to $\mathcal{O}^{ \pm}$.

YES, if $\mathcal{O}^{\prime}$ is equivalent to $\mathcal{O}^{ \pm}$; a map $\gamma \in O(V)$; a selection of points $\left\langle w_{k_{1}}\right\rangle, \ldots$, $\left\langle w_{k_{8}}\right\rangle$ with $\left\langle v_{i}\right\rangle \gamma=\left\langle w_{k_{i}}\right\rangle$ for $1 \leq i \leq 8$ if $\mathcal{O}^{+}=\mathcal{O} \gamma$, and $\left\langle u_{i}\right\rangle \gamma=\left\langle w_{k_{i}}\right\rangle$ if $\mathcal{O}^{-}=\mathcal{O} \gamma$.

Step 1. Compute $f\left(\mathcal{O}^{+}\right)$and $f\left(\mathcal{O}^{\prime}\right)$. If $f\left(\mathcal{O}^{+}\right) \neq f\left(\mathcal{O}^{\prime}\right)$, then go to END, the OUTPUT is NO. Otherwise go to Step 2.

Step 2. Compute the 8-tuple of fingerprints: $f_{i}=f\left(\mathcal{O}-\left\{\left\langle v_{1}\right\rangle, \ldots,\left\langle v_{i}\right\rangle\right\}\right)$ for $1 \leq i \leq 8$.

Step 3. Set $k_{1}=1$ if Step 3 is reached from Step 2. Otherwise $k_{1}$ is predefined. Compute $f_{k_{1}}^{\prime}=f\left(\mathcal{O}^{\prime}-\left\{\left\langle w_{k_{1}}\right\rangle\right\}\right)$. If $f_{1}=f_{k_{1}}^{\prime}$, go to Step 4. If $f_{1} \neq f_{k_{1}}^{\prime}$, increment $k_{1}:=k_{1}+1$, or if $k_{1}=126$ go to END and the OUTPUT is NO. (However in practice the last alternative never occurs as fingerprints already characterize the equivalence classes.)

Step 4. Choose a minimal $k_{2} \in\{1,2, \ldots, 126\}-\left\{k_{1}\right\}$ if Step 4 is reached from Step 3. Otherwise $k_{2}$ is predefined. Compute $f_{k_{2}}^{\prime}=f\left(\mathcal{O}^{\prime}-\left\{\left\langle w_{k_{1}}\right\rangle,\left\langle w_{k_{2}}\right\rangle\right\}\right)$. If $f_{2}=f_{k_{2}}^{\prime}$ go to Step 5. If $f_{2} \neq f_{k_{2}}^{\prime}$ increment $k_{2}:=k_{2}+1$, or if $k_{2}=126$ go to Step 3 and increment $k_{1}$.

Step 5. Choose a minimal $k_{3} \in\{1,2, \ldots, 126\}-\left\{k_{1}, k_{2}\right\}$ if Step 5 is reached from Step 4 . Otherwise $k_{3}$ is predefined. Compute $f_{k_{3}}^{\prime}=$ $f\left(\mathcal{O}^{\prime}-\left\{\left\langle w_{k_{1}}\right\rangle,\left\langle w_{k_{2}}\right\rangle,\left\langle w_{k_{3}}\right\rangle\right\}\right)$. If $f_{3}=f_{k_{3}}^{\prime}$, then select scalars $\alpha_{1}, \alpha_{2}, \alpha_{3}$ so 
that either $\operatorname{Gr}\left(v_{1}, v_{2}, v_{3}\right)=\operatorname{Gr}\left(\alpha_{1} w_{k_{1}}, \alpha_{2} w_{k_{2}}, \alpha_{3} w_{k_{3}}\right)$, or $\operatorname{Gr}\left(u_{1}, u_{2}, u_{3}\right)=$ $\operatorname{Gr}\left(\alpha_{1} w_{k_{1}}, \alpha_{2} w_{k_{2}}, \alpha_{3} w_{k_{3}}\right)$. Define a label $\varepsilon:=1$ or -1 according to whether the first or second case occurs. This label is never changed in higher level steps of the algorithm. Proceed with Step 6. If $f_{3} \neq f_{k_{3}}^{\prime}$, increment $k_{3}$ or return to Step 4 and proceed similarly as before.

Step 6. Choose a minimal $k_{4} \in\{1,2, \ldots, 126\}-\left\{k_{1}, k_{2}, k_{3}\right\}$ if Step 5 is reached from Step 4 . Otherwise $k_{4}$ is predefined. Compute $f_{k_{4}}^{\prime}=$ $f\left(\mathcal{O}^{\prime}-\left\{\left\langle w_{k_{1}}\right\rangle, \ldots,\left\langle w_{k_{4}}\right\rangle\right\}\right)$. If $f_{4}=f_{k_{4}}^{\prime}$ choose a scalar $\alpha_{4}$ if possible, so that $\operatorname{Gr}\left(v_{1}, \ldots, v_{4}\right)=\operatorname{Gr}\left(\alpha_{1} w_{k_{1}}, \ldots, \alpha_{4} w_{k_{4}}\right)$ if $\varepsilon=1$, or $\operatorname{Gr}\left(u_{1}, \ldots, u_{4}\right)=$ $\operatorname{Gr}\left(\alpha_{1} w_{k_{1}}, \ldots, \alpha_{4} w_{k_{4}}\right)$ if $\varepsilon=-1$. If such a scalar $\alpha_{4}$ can be found go to Step 7 . If this is not possible, or if $f_{4} \neq f_{k_{4}}^{\prime}$, increment $k_{4}$ or go back to Step 5 .

Step 7 to Step 10 are analogous to Step 6. Suppose we reach:

Step 10. And all the previous tests have succeeded. Then we have selected points $\left\{\left\langle w_{k_{1}}\right\rangle, \ldots,\left\langle w_{k_{8}}\right\rangle\right\}$ from $\mathcal{O}^{\prime}$; a label $\varepsilon= \pm 1$, and scalars $\alpha_{1}, \ldots, \alpha_{8}$. Next, we define an orthogonal map $\gamma$ by $v_{i} \gamma=\alpha_{i} w_{k_{i}}$ if $\varepsilon=1$, and $u_{i} \gamma=\alpha_{i} w_{k_{i}}$ if $\varepsilon=-1$ for $1 \leq i \leq 8$. Check whether $\mathcal{O}^{+} \gamma=\mathcal{O}^{\prime}$ or $\mathcal{O}^{-} \gamma=\mathcal{O}^{\prime}$, respectively. In either case the OUTPUT is YES together with $\varepsilon, \gamma$, and $w_{k_{1}}, \ldots, w_{k_{8}}$. Otherwise increment $k_{8}$, or go to Step 9. (It was found that once Step 10 is reached the test is always positive).

END.

To summarize: The fingerprint and the Gram matrix are used to sieve out those 8-tuples from $\mathcal{O}^{\prime}$ which are suitable for the construction of an equivalence map. The equivalence test can be modified in an obvious way to compute a set of generators for the automorphism group of an ovoid - which can be represented either as permutations on 126 points, or as elements of $G O(V)$-and to compute its order (see [3, 7]).

\section{Starter SETS}

Let $\mathcal{O}$ be an ovoid in $V$ and $P$ an isotropic point in $V$ not belonging to $\mathcal{O}$. Set $\bar{P}=$ $P^{\perp} / P$ and $\mathcal{O}_{P}=\left\{\bar{X} \mid X \in \mathcal{O} \cap P^{\perp}\right\}$, where $\bar{X}=(X+P) / P$. Then $\mathcal{O}_{P}$ is an ovoid in $\bar{P}$, which is an orthogonal space of type $\Omega^{+}(6, q)$. As we mentioned previously, ovoids in $\Omega^{+}(6, q)$ correspond via the Klein correspondence to the translation planes of order $q^{2}$. For $q=5$, there are according to Czerwinski and Oakden [5] precisely 21 equivalence classes of such ovoids. We denote these as $\mathcal{P}_{1}, \ldots, \mathcal{P}_{21}$ (cf. [2]). The linear span of each of the $\mathcal{P}_{1}, \ldots, P_{20}$ is always the 6-dimensional space $\Omega^{+}(6,5)$, while the ovoid $\mathcal{P}_{21}$ corresponds to the desarguesian plane. Thus $\left\langle\mathcal{P}_{21}\right\rangle$ is a subspace of type $\Omega^{-}(4,5)$ in $\Omega^{+}(6,5)$, and $\mathcal{P}_{21}$ is the set of isotropic points of this subspace.

By [5] we know that $\mathcal{O}_{P}$ must be equivalent to one of the $\mathcal{P}_{i}$. Choose a set of $m$ maximaly linearly independent points: $\bar{X}_{1}, \ldots, \bar{X}_{m} \in \mathcal{O}_{P}$; i.e. $m=6$ for $\mathcal{P}_{i}$ if $i<21$ and $m=4$ for $\mathcal{P}_{21}$. Choose any preimages $X_{1}, \ldots, X_{m}$ or $X_{1}^{\prime}, \ldots, X_{m}^{\prime}$ in $P^{\perp}$. Clearly $\left\langle X_{1}, \ldots, X_{m}\right\rangle$ and $\left\langle X_{1}^{\prime}, \ldots, X_{m}^{\prime}\right\rangle$ can be mapped onto each other by an isometry which fixes $P$ and takes $X_{i} \mapsto X_{i}^{\prime}$. Thus we can choose generators $X_{1}=\left\langle x_{1}\right\rangle, \ldots, X_{m}=\left\langle x_{m}\right\rangle$ and work with these.

If $\bar{X} \in \mathcal{O}_{P}-\left\{\bar{X}_{1}, \ldots, \bar{X}_{m}\right\}$ and $P=\langle p\rangle$, then the preimage of $\bar{X}$ in $\mathcal{O}$ is $\langle x+\alpha p\rangle$ for some $\alpha \in G F(5) ; x \in R=\left\langle x_{1}, \ldots, x_{m}\right\rangle$ where the $x$ are chosen in some canonical way - say by normalizing the first nonzero coefficient for the $x_{i}$ 's to 1 . Note that if $\alpha \neq 0$, there is an isometry which fixes $R$ element-wise and takes $\alpha p$ onto $p$. Thus we may write the preimage $X_{m+1}$ of $\bar{X}_{m+1} \in \mathcal{O}_{P}-\left\{\bar{X}_{1}, \ldots, \bar{X}_{m}\right\}$ as either 
$\left\langle x_{m+1}\right\rangle$, or $\left\langle x_{m+1}+p\right\rangle$ with $x_{m+1} \in R$. Similarly, $\bar{X}_{m+2} \in \mathcal{O}_{P}-\left\{\bar{X}_{1}, \ldots, \bar{X}_{m+1}\right\}$ has preimages $\left\langle x_{m+2}\right\rangle$, or $\left\langle x_{m+2}+p\right\rangle$ if $X_{m+1} \subset R$. If however $X_{m+1}=\left\langle x_{m+1}+p\right\rangle$ we have no choice but to consider all the cases of $X_{m+2}=\left\langle x_{m+2}+\alpha p\right\rangle$, where $\alpha \in G F(5)$ as possible candidates of a preimage. We use these observations to produce starter sets of size $m+3$, which have the property that every ovoid in $V$ contains up to equivalence starter sets of the given types.

Let $R$ denote a subspace either of type $\Omega^{+}(6,5)$ or $\Omega^{-}(4,5)$, and $P=\langle p\rangle \subset R^{\perp}$ an isotropic point. Let $\mathcal{P}_{k}=\left\{X_{i}=\left\langle x_{i}\right\rangle \mid 1 \leq i \leq 26\right\}$ be an ovoid as described above. Define $R$ to be $\left\langle x_{1}, \ldots, x_{6}\right\rangle$ if $k<21$, and $R=\left\langle x_{1}, \ldots, x_{4}\right\rangle$ (of type $\Omega^{-}(4,5)$ ) if $k=21$.

Then each $\mathcal{P}_{k}$ gives rise to 32 starter sets of the form $\left\{X_{1}, \ldots, X_{m}, Y_{m+1}\right.$, $\left.Y_{m+2}, Y_{m+3}\right\}$. Norming from the left and setting $X_{k}^{\alpha}=\left\langle x_{k}+\alpha p\right\rangle$ for $\alpha \in G F(5)$ (i.e. $\left.X_{k}=X_{k}^{0}\right)$, we see that $\left(Y_{m+1}, Y_{m+2}, Y_{m+3}\right)$ ranges over the following 32 triples:

(i) $\left(X_{m+1}^{0}, X_{m+2}^{0}, X_{m+3}^{a}\right), a=0,1$,

(ii) $\left(X_{m+1}^{0}, X_{m+2}^{1}, X_{m+3}^{a}\right), a \in G F(5)$, and

(iii) $\left(X_{m+1}^{1}, X_{m+2}^{a}, X_{m+3}^{b}\right), a, b \in G F(5)$.

Thus each $\mathcal{P}_{k}$, for $k<21$ gives 32 starter sets of length 9 , while $\mathcal{P}_{21}$ gives 32 starter sets of length 7 .

Lemma 2. Up to equivalence any ovoid in $V$ contains one of the 672 starter sets of the type (i), (ii), or (iii) defined above.

We first compute all the completions of the 640 starter sets of length 9. The completion algorithm is described in Section [5. In the remaining case of the 32 starter sets of length 7 further reductions are possible, as we shall now explain.

An ovoid $\mathcal{O}$ not found in the first 640 cases has the property that for every isotropic point $P \notin \mathcal{O}$ the projection $\mathcal{O}_{P}$ is of type $\mathcal{P}_{21}$. Thus if $\mathcal{L}=\left\{\left\langle v_{1}\right\rangle, \ldots,\left\langle v_{m}\right\rangle\right\}$ is the list of all isotropic points and if $\mathcal{S}=\left\{\left\langle y_{1}\right\rangle, \ldots,\left\langle y_{7}\right\rangle\right\}$ is a starter set belonging to $\mathcal{P}_{21}$, we can remove all the candidates $\left\langle v_{i}\right\rangle$ from the list $\mathcal{L}$ which satisfy the following condition.

$(*)$ : There is a 4 -set $\left\{y_{i_{1}}, \ldots, y_{i_{4}}\right\}$ such that $\operatorname{Gr}\left(y_{i_{1}}, \ldots, y_{i_{4}}, v_{i}\right)$ is nonsingular.

Otherwise, for an isotropic point $Q$ in $\left\langle y_{i_{1}}, \ldots, y_{i_{4}}\right\rangle^{\perp}$, the ovoid $\mathcal{O}_{Q}$ is not of type $\mathcal{P}_{21}$. In fact we can make even further reductions in this case. The details are given in Section [6.

\section{Completions}

We identify $V$ with $G F(5)^{8}$ and choose a symplectic basis for $V$, i.e., the inner product is given by

$$
\left(\left(a_{1}, \ldots, a_{8}\right),\left(b_{1}, \ldots, b_{8}\right)\right)=\sum_{i=1}^{4}\left(a_{i} b_{i+4}+a_{i+4} b_{i}\right) .
$$

By abuse of notation we represent points by a generating vector. Clearly we may assume that an ovoid always contains $P_{0}=(0,0,0,0,0,0,0,1)$. Thus any other point $P=\left(x_{1}, \ldots, x_{8}\right)$ of this ovoid has $x_{4} \neq 0$, so that we can normalize this vector so that $x_{4}=1$. Let $\mathcal{L}$ be the set of 15625 isotropic points $\left(b_{1}, b_{2}, b_{3}, 1, b_{4}, b_{5}, b_{6}, c\right)$ of this form, where $c=-\left(b_{1} b_{4}+b_{2} b_{5}+b_{3} b_{6}\right)$. For a 3-tuple $\left(b_{1}, b_{2}, b_{3}\right) \in G F(5)^{3}$, let $\mathcal{L}\left(b_{1}, b_{2}, b_{3}\right)$ be the subset of $\mathcal{L}$ whose first 3 coordinates are $\left(b_{1}, b_{2}, b_{3}\right)$. Then $\mathcal{L}$ is partitioned into 125 subsets of this form. Observe that for any $P, P^{\prime} \in \mathcal{L}\left(b_{1}, b_{2}, b_{3}\right)$, 
it is always the case that the inner product $\left(P, P^{\prime}\right)$ is zero. Thus an ovoid which contains $P_{0}$ intersects each of these subsets in precisely one point. Rename the sets $\mathcal{L}\left(b_{1}, b_{2}, b_{3}\right)$ as $\mathcal{L}_{1}, \ldots, \mathcal{L}_{125}$. Assume that $\mathcal{S}=\left\{P_{0}, P_{1}, \ldots, P_{k}\right\}$ is a starter set such that $P_{i} \in \mathcal{L}_{i}$ for $1 \leq i \leq k$. To complete $\mathcal{S}$ to an ovoid we have to pick a $P_{j} \in \mathcal{L}_{j}$ for $k+1 \leq j \leq 125$ such that $\left(P_{i}, P_{j}\right) \neq 0$ for all $0 \leq i<j \leq 125$. This is accomplished by the following three steps.

Step 1. Reduce $\mathcal{L}_{k+1}, \ldots, \mathcal{L}_{125}$ to subsets $\mathcal{L}_{k+1}^{\prime}, \ldots, \mathcal{L}_{125}^{\prime}$ such that $\left(P_{i}, P\right) \neq 0$ for $1 \leq i \leq k, P \in \mathcal{L}_{k+1}^{\prime} \cup \cdots \cup \mathcal{L}_{125}^{\prime}$

Step 2. Form a $(125-k)$-partite graph $\Gamma$ whose vertices correspond to the points of $\mathcal{L}_{k+1}^{\prime} \cup \cdots \cup \mathcal{L}_{125}^{\prime}$. Two vertices $X \in \mathcal{L}_{i}^{\prime}$ and $Y \in \mathcal{L}_{j}^{\prime}, i \neq j$ are joined by an edge in $\Gamma$, if and only if $(X, Y) \neq 0$.

Step 3. Enumerate all the complete subgraphs of $\Gamma$ of size $125-k$.

The complete subgraphs of $\Gamma$ together with $\mathcal{S}$ are all the completions of $\mathcal{S}$. For the enumeration in Step 3, we used the same straightforward algorithm which we previously used in [3] and 7]. For the reader's convenience we outline this algorithm as follows.

If a partial completion of $\mathcal{S}$ has reached stage $m$, i.e., it has the form $\left\{P_{0}, \ldots, P_{k}\right.$, $\left.\ldots, P_{m}\right\}$ with $P_{i} \in \mathcal{L}_{i}^{\prime}$, then the sets $\mathcal{L}_{m}^{\prime}, \ldots, \mathcal{L}_{125}^{\prime}$ have the reduced form $\mathcal{L}_{m}^{m}, \ldots$, $\mathcal{L}_{125}^{m}$. Now reduce $\mathcal{L}_{j}^{m}$ to $\mathcal{L}_{j}^{m+1}$ for $j \geq m+1$, such that $\mathcal{L}_{j}^{m+1}$ contains only those points $P$ with $\left(P, P_{m}\right) \neq 0$. If all the $\mathcal{L}_{j}^{m+1}$ are nonempty, choose $P_{m+1}$ as the first element in $\mathcal{L}_{m+1}^{m+1}$ and proceed to level $m+1$. Otherwise replace $P_{m} \in \mathcal{L}_{m}^{m}$ by its successor, or if this is not possible go back to level $m-1$.

\section{Computations}

Again let $\mathcal{L}$ be the list of 15625 isotropic points of type $(*, *, *, 1, *, *, *, *)$ and assume that the starter sets always contain the point $P_{0}$. The starter sets of size 9 originating from the ovoids $\mathcal{P}_{1}, \ldots, \mathcal{P}_{20}$ are reduced in Step 1 to a sublist $\mathcal{L}_{0}$ of size $2300-3000$ of compatible points. So that for $\mathcal{L}_{0}$ all the complete subgraphs of size 117 have to be found. The completions of the 32 starter sets belonging to $\mathcal{P}_{i}$ consumed between 2 and 18 hours. Not unexpectedly, the starter sets with $\left|\mathcal{L}_{0}\right|$ close to 3000 consumed the bulk of the computing time and produced most of the completions. The 640 cases produced a list $\mathcal{C}$ of 262 completions. The equivalence test then reduced $\mathcal{C}$ to a sublist $\mathcal{C}_{0}$ of three ovoids in a few minutes.

Computing the automorphism groups gave the orders 756000, 2903040, and 36228800 , establishing that $\mathcal{C}$ contained 220 ovoids of the unitary type, 30 ovoids of the symplectic type and 12 ovoids of the symmetric type. For each type of ovoid, we determined (cf. Section 3) the matrix generators of the groups which left the ovoid invariant. Using these generators we performed various computations with GAP [15]. This established independently that the groups listed in Table I did indeed have the given structure.

In the remaining case of the desarguesian ovoid $\mathcal{P}_{21}$, it was inconvenient to use starter sets of length 7 as indicated in Section 4 . Instead we proceeded as follows. Let $R \subset V$ be of type $\Omega^{-}(4,5), P=\langle p\rangle \subset R^{\perp}$ isotropic, and $\mathcal{P}_{21}=\left\{X_{i}=\left\langle x_{i}\right\rangle \mid 1 \leq\right.$ $i \leq 26\}$ the isotropic points in $R$ such that $R=\left\langle x_{1}, \ldots, x_{4}\right\rangle$.

First, in accordance with condition $(*)$ of Section 4 , all the $\left\langle v_{i}\right\rangle \in \mathcal{L}$ with $\operatorname{Gr}\left(x_{1}, \ldots, x_{4}, v_{i}\right) \neq 0$ were removed, producing a sublist $\mathcal{L}_{0}$ of size 2625 .

Next we checked that a 5 -starter set of the form $\widetilde{\mathcal{S}}_{k}=\left\{X_{1}, \ldots, X_{4}, \widetilde{X}_{k}\right\}, \widetilde{X}_{k}=$ $\left\langle x_{k}+p\right\rangle, 5 \leq k \leq 17$, again reduces $\mathcal{L}_{0}($ by $(*))$, to a list $\mathcal{L}_{00}$ of 125 elements. 
And of course no completions were found. Thus any ovoid which projects only onto $\mathcal{P}_{21}$ must contain up to equivalence a starter set $\mathcal{S}=\left\{X_{1}, \ldots, X_{17}\right\}$. However, $\mathcal{S}$ reduces $\mathcal{L}_{0}$ to a list of almost (surprisingly) 1000 candidates and no completions were found.

\section{Final REMARKS}

A. The classification of the 8-dimensional ovoids yields the following

Corollary 3. The ovoids in $\Omega^{+}(6,5)$ listed in Table II (corresponding to only some of the translation planes of order 25 (cf. [2], [5]), are the only projections of the 8-dimensional ovoids.

The statement that every ovoid in $\Omega^{+}(8,5)$ has a basis is equivalent to the fact that there are no ovoids in $\Omega(7,5)$. Hence it follows from our enumeration that:

Corollary 4. There are no ovoids in $\Omega(7,5)$.

The projections of the $W\left(E_{7}\right)$ and $S_{10}$ ovoids are given in 2].1. Thus we only have to consider the $P G U_{3}(5) \cdot 2$ ovoid. Starting with a set of generating matrices of $P G U_{3}(5) \cdot 2$ which leave this ovoid invariant, a GAP calculation established that $P G U_{3}(5) \cdot 2$ has 3 orbits on the set of 19656 isotropic points of $\Omega^{+}(8,5)$. These orbits have lengths 3780,15750 and 126 (cf. Theorems 4.5 and 4.6 of [10]). The isotropic vectors belonging to the orbit of length 126 constitute the ovoid, leaving just two candidate orbits for projection. Isotropic points belonging to the orbit of length 3780 project on to the ovoid whose fingerprint is $\Xi_{6}$, while the orbit of length 15750 projects onto the ovoid with fingerprint $\Xi_{9}$.

A double entry in the last column of Table II indicates that the 6-dimensional ovoid is the projection of two 8-dimensional ovoids; i.e. there is an isotropic point belonging to a $W\left(E_{7}\right)$ orbit and a $S_{10}$ orbit, which projects $\mathcal{O}_{E}$ and $\mathcal{O}_{S}$ onto equivalent 6 -dimensional ovoids. The fourth column refers to the orbit lengths of the automorphism groups of the 6-dimensional ovoids (see [2, 6]).

In particular, Corollary 3 gives a negative answer to the question, in odd characteristic, whether or not every ovoid in $\Omega^{+}(6, q)$ is a projection of an 8-dimensional ovoid. (This question was posed by W. M. Kantor.) The ovoids in $\Omega^{+}(6,2)$ and $\Omega^{+}(6,3)$ have this property (see [10]). In even characteristic, $\Omega^{+}(6,4)$ is a counterexample; this follows from [8, 10, 9]. Corollary 3 also shows that the $r$-ary variant $\mathcal{O}_{r, 5}(\mathbf{x})$ of the $E_{8}$ root lattice construction of Moorhouse [12] does not produce any new ovoids for any value of $r$.

TABLE II. Projections of ovoids in $\Omega^{+}(8,5)$.

\begin{tabular}{ll|l|l|l} 
& Fingerprints & {$[5$, 6] } & Orbits & $\Omega^{+}(8,5)$ Ovoids \\
\hline$\Xi_{1}:$ & $80^{0}, 12^{480}, 20^{80}, 24^{10}, 25^{26}$ & $B_{5}$ & 10,16 & $\mathcal{O}_{S}, \mathcal{O}_{E}$ \\
$\Xi_{2}:$ & $4^{420}, 8^{180}, 12^{20}, 24^{30}, 25^{26}$ & $B_{4}$ & 6,20 & $\mathcal{O}_{S}, \mathcal{O}_{E}$ \\
$\Xi_{3}:$ & $0^{20}, 4^{360}, 8^{240}, 16^{18}, 24^{12}, 25^{26}$ & $S_{2}$ & 6,20 & $\mathcal{O}_{E}$ \\
$\Xi_{5}:$ & $0^{36}, 4^{288}, 8^{180}, 12^{80}, 16^{42}, 20^{24}, 25^{26}$ & $S_{5}$ & 8,18 & $\mathcal{O}_{S}, \mathcal{O}_{E}$ \\
$\Xi_{6}:$ & $0^{50}, 4^{200}, 8^{100}, 12^{200}, 16^{100}, 25^{26}$ & $A_{3}$ & 1,25 & $\mathcal{O}_{U}$ \\
$\Xi_{9}:$ & $0^{144}, 4^{336}, 8^{168}, 24^{2}, 25^{26}$ & $A_{8}$ & $1,1,24$ & $\mathcal{O}_{U}$ \\
$\Xi_{11}:$ & $0^{192}, 4^{432}, 8^{24}, 24^{2}, 25^{26}$ & $A_{2}$ & 2,24 & $\mathcal{O}_{E}$
\end{tabular}

\footnotetext{
${ }^{1}$ The names of the planes in Table 2 of [2] are those used in the preprint versions of [5] and [6].
} 
B. How reliable is such a computer-aided enumeration? First, we observe that if any of the 32 starter sets belonging to an ovoid $\mathcal{P}_{i}$ failed to produce a completion, then none of the 8-dimensional ovoids could be projected onto $\mathcal{P}_{i}$. Secondly, if our enumeration had gaps, one would expect, in view of the distribution of the three types of completions in the list $\mathcal{C}$, that any such omitted ovoid should have a large automorphism group, say of order $\geq 1000000$. However, such a highly symmetric ovoid would almost certainly have been detected earlier by group theoretic means. In any case an independent verification of our result is desirable.

C. For the three 8-dimensional ovoids ovoid in $\Omega^{+}(8,5)$ the automorphism group Aut $(\mathcal{O})$ does not cover $G O(V) / O(V)$. This raises the following question: Are there 8-dimensional ovoids whose automorphism groups cover this quotient?

\section{ACKNOWLEDGMENT}

We would like to thank an anonymous referee for comments which led to improvements in our exposition.

\section{REFERENCES}

[1] A. Blokhuis and G. E. Moorhouse, Some $p$-ranks related to orthogonal spaces, J. Alg. Combinatorics 4 (1995), 295-316. MR 96g:51011.

[2] C. Charnes, Quadratic matrices and translation planes of order $5^{2}$, Coding Theory, Design Theory, Group Theory, Proceedings of the M. Hall Conference, eds. D. Jungnickel and S. A. Vanstone, Wiley, New York 1993, pp. 155-161. MR 94h:51016

[3] C. Charnes and U. Dempwolff, The translation planes of order 49 and their automorphism groups, Math. Comp. 67 (1998), 1207-1224. MR 98g:51007]

[4] J. H. Conway, P. B. Kleidman and R. A. Wilson, New families of ovoids in $\Omega_{8}^{+}$, Geom. Ded. 26 (1988), 157-170. MR 89h:51014

[5] T. Czerwinski and D. Oakden, The translation planes of order twenty-five, J. Comb. Theory (A) 59 (1992), 193-217. MR 93c:51009

[6] T. Czerwinski, The collineation groups of the translation planes of order 25, Geom. Ded. 39 (1991), 125-137. MR 92i:51013

[7] U. Dempwolff, Translation planes of order 27, Designs, Codes and Crypt. 4 (1994), 105-121. MR 95a:51012

[8] U. Dempwolff and A. Reifart, The classification of the translation planes of order 16 I, Geom. Ded. 15 (1983), 137-153. MR 86d:51006

[9] A. Gunawardena, Classification of ovoids in $\Omega_{8}^{+}(4)$, submitted for publication, 1996.

[10] W. M. Kantor, Ovoids and translation planes, Can. J. Math. 34 (1982), 1195-1207. MR 84b:51019

[11] W. M. Kantor, Spreads, translation planes and Kerdock sets I, SIAM J. Alg. Discr. Math. 3 (1982), 151-165. MR 83m:51013b

[12] G. E. Moorhouse, Ovoids from the $E_{8}$ root lattice, Geom. Ded. 46 (1993), 287-297. MR 94d:51011

[13] G. Mason and E. E. Shult, The Klein correspondence and the ubiquity of certain translation planes, Geom. Ded. 21 (1986), 29-50. MR 87g:51002

[14] R. Mathon and G. Royle, The translation planes of order 49, Designs, Codes and Crypt. 5 (1995), 57-72. MR 95j:51016

[15] M. Schönert et al., GAP Groups, Algorithms and Programming 3.4.4, Lehrstuhl D für Mathematik, RWTH Aachen, 1997.

[16] E. E. Shult, Nonexistence of ovoids in $\Omega_{10}^{+}(3)$, J. Comb. Theory (A) 51 (1989), 250-257. MR 90f:51015

[17] J. A. Thas, Ovoids and spreads of finite classical polar spaces, Geom. Ded. 10 (1981), 135-144. MR 82g:05031 
Department of Computer Science, University of Melbourne, Parkville, ViC 3052 Australia

E-mail address: charnes@cs.mu.oz.au

FB Mathematik Universität Kaiserslautern, Postfach 3049, 67653 Kaiserslautern, GERMANY

E-mail address: dempwolff@mathematik.uni-kl.de 